\title{
Depressão e relação com idade em cuidadores de familiares portadores de síndrome demencial
}

\author{
Josiane Pawlowski \\ Tonantzin Ribeiro Gonçalves \\ Juliana Balbinot Hilgert \\ Fernando Neves Hugo \\ Mary Clarisse Bozzetti \\ Denise Ruschel Bandeira \\ Universidade Federal do Rio Grande do Sul
}

\begin{abstract}
Resumo
Avaliou-se a presença de sintomas depressivos em cuidadores de familiares portadores de síndrome demencial comparados a não cuidadores e a relação deste desfecho em três faixas etárias. Participaram 84 cuidadores e 101 não cuidadores que residiam na região metropolitana de Porto Alegre, RS, Brasil. Aplicou-se um questionário de dados sociodemográficos e o Inventário Beck de Depressão. Os cuidadores revelaram níveis mais elevados de sintomas depressivos e com maior gravidade nos aspectos cognitivos e afetivos do inventário de depressão quando comparados aos não cuidadores. Foram encontrados níveis mais baixos de sintomas de depressão em indivíduos de maior faixa etária (65 a 83 anos). São discutidas as limitações do inventário na avaliação de sintomas de depressão em pessoas idosas. Ressalta-se a necessidade de suporte psicológico e apoio social aos cuidadores de familiares com síndrome demencial.
\end{abstract}

Palavras-chave: depressão; idade; cuidadores; demência.

\begin{abstract}
Depression related to age in family caregivers of patients with dementia. Depression symptoms related to age in family caregivers of dementia patients compared to non-caregivers were evaluated. Eighty-four family caregivers to dementia patients and one hundred and one non-caregivers that lived in Porto Alegre (Brazil) region participated of this study. They answered a sociodemographic questionnaire and the Beck Depression Inventory. The caregivers presented higher degree of depression symptoms and higher degree of cognitive and affective aspects of depression inventory those non-caregivers. Lowest degree of depression symptoms were presented by the oldest participants. Limitations of the inventory to evaluate depression in older people were discussed. The study emphasizes the necessity of psychological and social support to family caregivers of dementia patients.
\end{abstract}

Keywords: depression; age; caregivers; dementia.

$\mathrm{A}$ depressão é uma das doenças psiquiátricas a qual muitos pesquisadores da área da saúde dedicam-se a investigar em razão de sua alta prevalência na população. Estudos epidemiológicos indicam características sociodemográficas, condições precárias de vida e eventos vitais estressantes entre os principais fatores de risco para a depressão. Dentre as características demográficas, a revisão realizada por Snowdon (2002) apontou que em torno de $10 \%$ da população idosa apresenta quadros depressivos com significância clínica. Esse índice desperta preocupação em função do impacto negativo na qualidade de vida, na saúde física e no desempenho cognitivo em pessoas nesta faixa etária (Penninx, Deeg, van Eijk, Beekman,
\& Guralnik, 2000; Schoevers et al., 2000).

Apesar da concordância quanto à alta incidência de depressão entre idosos, os estudos divergem quanto à faixa etária de maior prevalência. Em um estudo com idosos de Pelotas, a média de sintomas depressivos foi maior para indivíduos mais velhos (Gazalle, Lima, Tavares, \& Hallal, 2004). Outros pesquisadores encontraram uma prevalência menor de sintomas de depressão e ansiedade entre pessoas mais velhas em uma população de 2.622 australianos de 18 a 79 anos (Christensen et al., 1999). Jorm (2000) afirma que há uma tendência ao aumento dos sintomas por meio dos grupos etários seguida por uma queda. Tanto Gazalle et al. (2004) quanto Christensen et 
al. (1999) destacam que os sintomas físicos e psicológicos de depressão reportados por idosos são qualitativamente diferentes daqueles referidos por pessoas mais jovens. Para Gazalle et al. (2004), dentre as características clínicas peculiares dos quadros depressivos de idosos destacam-se a diminuição da resposta emocional e o predomínio de sintomas como a diminuição do sono, a perda de prazer nas atividades habituais, as ruminações sobre o passado e a perda de energia. Segundo Leite, Carvalho, Barreto, e Falcão (2006), as mulheres idosas também parecem ser mais vulneráveis a problemas de saúde mental, em particular a depressão.

Entre outros fatores de risco para depressão, possuir incapacidades físicas também tem destaque (Mann, 2001; Schoevers et al., 2000; Snowdon, 2002). Contudo, é importante lembrar que em idosos as queixas somáticas são bastante comuns e que isto pode trazer um viés à avaliação de sintomas de depressão (Trentini et al., 2005). Neste sentido, um estudo norueguês de base populacional com 60.869 pessoas com idades entre 20 e 89 anos demonstrou que, mesmo após o controle de múltiplas variáveis, permaneceu um efeito residual da idade sobre o aumento da depressão que não pôde ser explicado pelos prejuízos físicos comuns na velhice (dificuldades de visão, audição e movimento) e pela presença de doenças somáticas, fatores estes que contribuíram mais fortemente para a depressão entre os mais velhos (Stordal, Mykletun, \& Dahl, 2003).

Dentre os aspectos sociais envolvidos, viver sozinho, pobreza e baixa escolaridade são comumente relacionados à depressão (Gazalle et al., 2004; Snowdon, 2002; Stordal et al., 2003). A institucionalização, o isolamento social, a perda do cônjuge e as situações de estresse crônico também se mostram relevantes na precipitação de sintomas depressivos (Gazalle et al., 2004; Mann, 2001; Plati, Covre, Lukasova, \& Macedo, 2006; Schoevers et al., 2000).

No que diz respeito a situações de estresse crônico, pesquisas com familiares de portadores de síndrome demencial apontam o impacto psicológico que o cuidado prolongado e a deterioração progressiva do doente provocam no cuidador (Cacioppo et al., 1998; Gaugler, Davey, Pearlin, \& Zarit, 2000; Pinquart \& Sörensen, 2003; Russo, Vitaliano, Brewer, Katon, \& Becker, 1995; Vitaliano, Zhang, \& Scanlan, 2003). Na maior parte dos casos, o cuidador responsável é o cônjuge ou os filhos que residem na mesma casa que o doente (Pinquart \& Sörensen, 2003; Russo et al., 1995). Os estudos têm demonstrado elevados níveis de estresse, depressão e ansiedade dessa população quando comparada a grupos controles e/ou normas populacionais (Bandeira et al., 2007; Bauer et al., 2000; Cacioppo et al., 1998; Ferrara et al., 2008; Russo et al., 1995; Schoenmakers, Buntinx, \& De Lepeleire, 2009; Vitaliano, Russo, Young, Teri, \& Maiuro, 1991). Cuidar de um familiar doente também tem sido associado a problemas de saúde física, além de afetar a qualidade de vida (Bodnar \& Kiecolt-Glaser, 1994; Goode, Haley, Roth, \& Ford, 1998; Ho, Chan, Woo, Chong, \& Sham, 2009; Vitaliano et al., 2003).

Com relação aos sintomas de depressão, as escalas de autorrelato utilizadas indicam que mais de $30 \%$ destes cuidadores apresentam níveis de depressão de média a moderada (Vitaliano et al., 1991). O estudo de Russo et al. (1995) revela que a história prévia de depressão não explica o aumento dos índices de ocorrência destes transtornos nos cuidadores após o início da atividade de cuidado. Outros achados apontam para a continuidade da depressão após três anos da morte do doente, sugerindo o impacto do luto e da situação continuada de cuidado (Bodnar \& Kiecolt-Glaser, 1994). Por sua vez, Sanders e Adams (2005) encontraram associação da depressão com uma pior avaliação da própria saúde e o relato de sentimentos de luto, sobrecarga e isolamento.

A influência da relação de parentesco com o doente sobre a saúde emocional dos cuidadores diverge entre os estudos. Alguns pesquisadores encontraram níveis de depressão e ansiedade semelhantes entre cuidadores cônjuges e filhos (Anthony-Bergstone, Zarit, \& Gatz, 1988; Dura, Stukenberg, \& Kiecolt-Glaser, 1991). Por outro lado, o estudo de meta-análise de Pinquart e Sörensen (2003) apontou que as diferenças de estresse e depressão entre cuidadores e não cuidadores eram significativamente maiores em amostras de cônjuges do que em amostras de filhos. Estudos mais recentes mostraram maior sobrecarga psicológica entre os filhos que tendem a acumular tarefas com o familiar doente e com a própria família (CondeSala, Garre-Olmo, Turró-Garriga, Vilalta-Franch, \& LópezPousa, no prelo; Yeager, Hyer, Hobbs, \& Coyne, 2010).

Assim como estudos com a população em geral, os estudos com cuidadores também se mostram divergentes no que se refere à frequência de depressão e à relação entre idade e gravidade dos sintomas depressivos nesta população. Pinquart e Sörensen (2003) destacaram que as diferenças entre os níveis de depressão de cuidadores e não cuidadores foram maiores em amostras mais velhas. Já Sanders e Adams (2005) indicam que cuidadores mais jovens referem maiores níveis de depressão do que os mais velhos.

Considerando a situação de cuidado como geradora de estresse crônico e como um fator de risco para depressão, o objetivo deste estudo foi avaliar a presença de sintomas depressivos em cuidadores de familiares com síndrome demencial comparados a não cuidadores e examinar a relação desse desfecho em diferentes faixas etárias. Também foi objetivo deste estudo comparar a presença de diferentes características de depressão entre cuidadores e não cuidadores de diferentes faixas etárias e avaliar a presença de sintomas depressivos em cuidadores com diferentes graus de parentesco com a pessoa cuidada.

\section{Método}

\section{Participantes}

Participaram do estudo 84 cuidadores de familiares com síndrome demencial e 101 não cuidadores residentes na região metropolitana de Porto Alegre. Os participantes foram avaliados durante um projeto interdisciplinar que incluiu avaliações psicológica e odontológica. O referido projeto foi aprovado pelo Comitê de Ética em Pesquisa do Hospital de Clínicas de Porto Alegre (processo no 02304). Para a realização do presente artigo, do total de 274 participantes (129 cuidadores e 145 não 
cuidadores) avaliados no estudo interdisciplinar, foram excluídos 84 que faziam uso de psicofármacos e 5 casos com resultados extremos em depressão (outliers), visto que os resultados destes participantes poderiam provocar um viés nas análises.

Do total de 185 participantes deste estudo, 82,8\% são mulheres, com idade média de 61,68 anos $(D P=8,69)$, distribuídos uniformemente nos níveis de escolaridade (Fundamental, Médio e Superior) e com renda mensal média de $\mathrm{R} \$ 2.179,78(D P=$
1.726,86). As características sociodemográficas de cuidadores e não cuidadores separadamente são apresentadas na Tabela 1 . Não foi encontrada diferença significativa entre os grupos quanto à idade e à renda mensal. Contudo, os grupos diferiram quanto ao estado civil $\left(\chi^{2}=23,09 ; g l=3 ; p<0,0001\right)$.

Em relação ao grupo de cuidadores, $48,8 \%$ eram filhos do doente, $36,9 \%$ eram cônjuges e 14,3\% tinham algum outro grau de parentesco (genro/nora, irmão/ã, sobrinho/a e cunhado/a).

Tabela1

Características sociodemográficas por grupo

\begin{tabular}{|c|c|c|c|c|}
\hline \multirow{3}{*}{ Características } & \multicolumn{4}{|c|}{ Grupos } \\
\hline & \multicolumn{2}{|c|}{ Cuidadores } & \multicolumn{2}{|c|}{ Não cuidadores } \\
\hline & - & $\%$ & - & $\%$ \\
\hline$N$ & 84 & & 101 & \\
\hline \multicolumn{5}{|l|}{ Sexo $(n)$} \\
\hline Feminino & 65 & 77,4 & 88 & 87,1 \\
\hline Masculino & 19 & 22,6 & 13 & 12,9 \\
\hline \multicolumn{5}{|l|}{ Idade (anos) } \\
\hline$M(D P)$ & $\begin{array}{l}60,55 \\
(9,34)\end{array}$ & & $\begin{array}{l}62,62 \\
(8,05)\end{array}$ & \\
\hline \multicolumn{5}{|l|}{ Escolaridade $(n)$} \\
\hline Nível Fundamental & 30 & 35,7 & 36 & 35,6 \\
\hline Nível Médio & 24 & 28,6 & 28 & 27,0 \\
\hline Nível Superior & 30 & 35,7 & 37 & 36,6 \\
\hline \multicolumn{5}{|l|}{ Estado civil $(n)^{*}$} \\
\hline Solteiro & 10 & 11,9 & 9 & 8,9 \\
\hline Casado ou com companheiro & $62^{\mathrm{a}}$ & 73,8 & $46^{\mathrm{a}}$ & 45,5 \\
\hline Divorciado & 7 & 8,3 & 14 & 13,9 \\
\hline Viúvo & $5^{\mathrm{b}}$ & 6,0 & $32^{\mathrm{b}}$ & 31,7 \\
\hline \multicolumn{5}{|l|}{ Renda familiar (R\$) } \\
\hline$M(\mathrm{DP})$ & $\begin{array}{c}2.256,36 \\
(1.776,59)\end{array}$ & & $\begin{array}{c}2.115,45 \\
(1.690,25)\end{array}$ & \\
\hline
\end{tabular}

Notas. Teste de Qui-Quadrado de Pearson, ${ }^{*} p<0,001$.

${ }^{\mathrm{a}, \mathrm{b}}$ Para letras iguais, diferença estatisticamente significativa.

Entre os filhos cuidadores, 53,7\% eram casados ou possuíam companheiro. Em média, os familiares cuidavam do doente 16 horas por dia $(M=15,9 ; D P=8,0)$ ao longo de mais de quatro anos $(M=4,7 ; D P=3,5)$. A maioria dos familiares $(76,8 \%)$ dedicava-se ao cuidado por 12 horas diárias ou mais. Além disso, em torno de $70 \%$ deles participavam ou já tinham frequentado alguma vez um grupo de apoio, em especial os indivíduos com mais de 65 anos (74,6\% destes participavam de algum grupo) e $63 \%$ declararam receber auxílio de outras pessoas no cuidado.

Os cuidadores foram convidados a participar do estudo por meio de grupos de apoio para familiares de portadores de Alzheimer com sedes em dois hospitais universitários de Porto Alegre e por anúncios em jornais locais. Os critérios para inclusão dos cuidadores no estudo foram: a) ter vínculo familiar com o doente em qualquer grau; b) diagnóstico declarado pelo cuidador de síndrome demencial; c) responsabilizar-se por parte ou pela totalidade das atividades de cuidado do doente (cuidadores primários e secundários). Todos os cuidadores identificavam a si mesmos como cuidadores de um portador de síndrome demencial e, em sua grande maioria, relataram que o familiar possuía a doença de Alzheimer.

Os participantes do grupo de comparação (não cuidadores) foram recrutados por meio de programas de ensino e recreação para idosos ligados à universidade e a outras entidades comunitárias $(52,43 \%)$, por conveniência e por anúncios em jornais. O critério de participação consistiu em não ser responsável, total ou parcialmente, pelo cuidado de adultos e crianças incapacitados física ou mentalmente.

\section{Instrumentos e procedimentos}

Os participantes assinaram um Termo de Consentimento Livre e Esclarecido declarando o conhecimento de todas as etapas de investigação e consentindo sua participação. Para a avaliação psicológica do estudo geral associado à odontologia, 
responderam a um questionário de dados sociodemográficos e foram submetidos à avaliação psicológica por meio de escalas de depressão, ansiedade, desesperança e estresse. No presente estudo, foram analisados os resultados do Inventário Beck de Depressão - BDI ${ }^{1}$ (Beck \& Steer, 1993), adaptado e validado para o Brasil por (Cunha, 2001), que apresentou bom índice de consistência interna $(\alpha=0,87)$.

Com o objetivo de aprofundar a análise do instrumento, a escala foi avaliada em seus dois fatores: Cognitivo-Afetivo (itens 1 a 13) e Somático-Desempenho (itens 14 a 21). O fator Cognitivo-Afetivo inclui itens de autoavaliação sobre estados emocionais de irritação, sentimentos de desprazer, decepção, tristeza, fracasso, culpa e ideias de suicídio. O fator SomáticoDesempenho compreende as impressões sobre mudanças relacionadas à aparência física, saúde, hábitos alimentares e de sono, desempenho no trabalho e interesse sexual.

\section{Procedimentos para análise de dados}

Com o objetivo de avaliar a relação entre sintomas depressivos de cada grupo (cuidadores e não cuidadores) e a variável sociodemográfica escolaridade, aplicou-se a análise de variância ANOVA para comparar os resultados do BDI entre os três níveis de escolaridade da amostra. Para verificar a relação entre sintomas de depressão e as variáveis renda e idade, realizou-se o teste de correlação de Pearson entre os escores do BDI e estas variáveis sociodemográficas. Para comparar a média de sintomas de depressão e dos dois fatores do BDI entre cuidadores e não cuidadores, realizou-se a análise de Teste $t$ para amostras independentes.

A análise de variância ANOVA com post hoc Scheffé foi aplicada para comparar a média de sintomas de depressão de cuidadores, não cuidadores e amostra total em três diferentes faixas etárias. Para avaliar diferenças entre cuidadores e não cuidadores nos resultados das subescalas do BDI em cada faixa etária examinada, os dados foram analisados mediante Teste $t$ para amostras independentes. Também foi aplicada uma análise de variância ANOVA para comparar os resultados de média de sintomas de depressão e dos dois fatores do BDI em cuidadores agrupados em três tipos de relação de parentesco com o doente: cônjuge, filhos e outros.

\section{Resultados}

$\mathrm{Na}$ avaliação da relação entre sintomas depressivos e variáveis sociodemográficas, as médias dos escores do BDI não apresentaram diferença estatisticamente significativa quando comparados os três níveis de escolaridade da amostra. Também não foi encontrada correlação significativa entre renda e escores do BDI. Para a amostra total, a média no BDI foi de 10,15 (DP= $6,96)$, sendo maior $(t=3,62 ; g l=183 ; p<0,001)$ para o grupo de cuidadores $(M=12,12 ; D P=7,45)$ quando comparados aos não cuidadores $(M=8,51, D P=6,09)$. Os resultados da comparação de cuidadores e não cuidadores nos dois fatores ou subescalas do BDI indicou diferença estatisticamente significativa $(t=3,88$; $g l=157,64 ; p<0,001)$ na subescala Cognitivo-Afetivo, em que os cuidadores apresentaram escores mais altos $(M=6,87 ; D P=$ $4,98)$ que os não cuidadores $(M=4,26 ; D P=3,98)$. Na subescala Somático-Desempenho, os grupos não apresentaram diferenças estatisticamente significativas.

$\mathrm{Na}$ avaliação entre idade e sintomas depressivos, foi encontrada correlação estatística significativa entre a idade e os escores do BDI $(r=-0,30 ; p<0,001)$. Para análise mais detalhada desta relação, a amostra foi dividida em três faixas etárias (40-55, 56-64 e 65-83 anos), levando-se em consideração a distribuição da variável idade nesta amostra e aspectos do desenvolvimento do adulto. Conforme a Tabela 2, os participantes das faixas etárias de menor idade apresentaram níveis mais elevados de depressão. Para cuidadores, foi encontrada diferença estatística significativa $(p<0,05)$ entre as médias de sintomas depressivos do grupo de 56 a 64 anos de idade e o de idosos (65-83 anos). Já para não cuidadores, foi encontrada diferença estatística significativa ( $p$ $<0,05)$ entre as médias de sintomas depressivos do grupo mais jovem (40-55 anos) e o de idosos (65-83 anos).

Analisando os dois fatores ou subescalas do BDI separadamente (Figura 1), na subescala Somático-Desempenho os maiores escores mantiveram-se para os cuidadores das

Tabela 2

Média, desvio-padrão e resultados da Análise de variância em BDI de cuidadores, não cuidadores e total da amostra por faixa etária

\begin{tabular}{|c|c|c|c|c|c|c|c|c|}
\hline \multirow{3}{*}{ Grupo } & \multicolumn{6}{|c|}{ Faixa etária (anos) } & \multirow{3}{*}{$F$} & \multirow{3}{*}{$p$} \\
\hline & \multicolumn{2}{|r|}{$40-55$} & \multicolumn{2}{|r|}{$56-64$} & \multicolumn{2}{|r|}{$65-83$} & & \\
\hline & $n$ & $M(D P)$ & $n$ & $M(D P)$ & $n$ & $M(D P)$ & & \\
\hline Cuidadores & 31 & $13,45(7,48)^{a, b}$ & 22 & $14,73(7,58)^{\mathrm{a}}$ & 31 & $8,94(6,32)^{b}$ & 5,15 & $<0,01$ \\
\hline Não cuidadores & 23 & $12,30(6,88)^{\mathrm{a}}$ & 38 & $8,76(5,84)^{a, b}$ & 40 & $6,10(4,64)^{b}$ & 8,80 & $<0,001$ \\
\hline Total & 54 & $12,96(7,19)^{a}$ & 60 & $10,95(7,09)^{\mathrm{a}}$ & 71 & $7,34(5,58)^{b}$ & 11,85 & $<0,001$ \\
\hline
\end{tabular}

faixas etárias de menor idade. Foi evidenciado um declínio com o aumento da idade tanto para cuidadores quanto para não cuidadores, mas não foram verificadas diferenças estatisticamente significativas entre estes dois grupos nesta subescala quando comparados em cada uma das três faixas etárias. De maneira distinta, na subescala Cognitivo-Afetivo, foi encontrada diferença estatisticamente significativa entre cuidadores e não cuidadores nas faixas etárias de 56 a $64(t=$ $3,85 ; g l=58 ; p<0,001)$ e 65 a 83 anos $(t=2,4 ; g l=47,39 ; p$ $<0,05)$.

Especificamente em relação aos cuidadores, quando agrupados em relação aos três tipos de parentesco com o doente: 


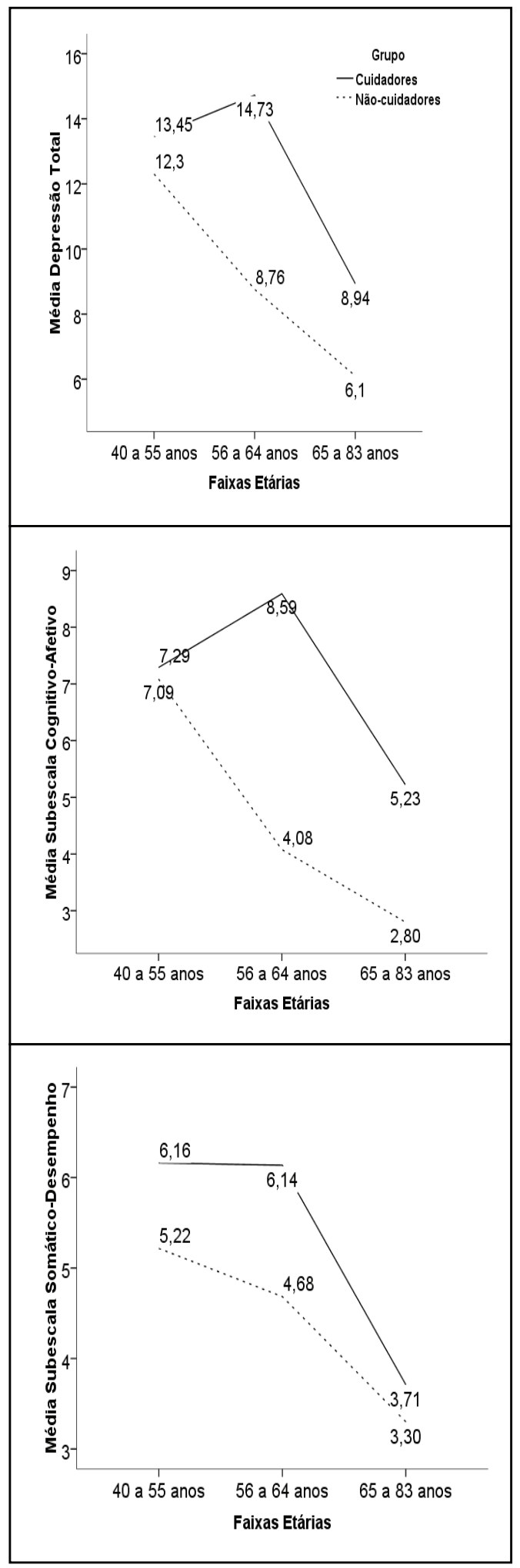

Figura 1. Média no escore total de depressão do BDI e nas subescalas Cognitivo-Afetivo e Somático-Desempenho do BDI para cuidadores e não cuidadores nas três faixas etárias.

cônjuge, filho e outros, não foram encontradas diferenças significativas nos resultados de média nas subescalas nem na escala total de depressão.

\section{Discussão}

A situação de cuidado de familiares portadores de síndrome demencial tem sido associada à presença de níveis mais elevados de sofrimento psicológico e de sintomas de estresse, ansiedade e depressão (Anthony-Bergstone et al., 1988; Bandeira et al., 2007; Russo et al., 1995; Vitaliano et al., 2003). No presente estudo, como foco principal, avaliou-se a presença de sintomas depressivos em cuidadores de familiares com síndrome demencial comparados a não cuidadores e foi examinada a relação desse desfecho em diferentes faixas etárias. Também foram comparadas duas facetas da depressão entre cuidadores e não cuidadores de diferentes faixas etárias. Além disso, buscou-se avaliar a influência de variáveis sociodemográficas em sintomas depressivos e a relação entre tais sintomas e o grau de parentesco com o doente, cujos resultados das análises serão discutidos nesta seção.

No que se refere às variáveis sociodemográficas, não foram encontradas diferenças para sintomas depressivos em cuidadores na comparação entre três níveis de escolaridade e não foi encontrada relação entre renda e depressão. Alguns autores revelam resultados contraditórios em relação às influências destas variáveis sobre sintomas de depressão avaliados em cuidadores. Dura, Stukenberg, e Kiecolt-Glaser (1990) encontraram que a renda e o nível educacional foram mais baixos para cuidadores com transtorno depressivo. Por outro lado, Russo et al. (1995) apontam a inexistência de relação entre renda e depressão. Apesar de não especificamente à depressão, em estudo com amostra brasileira de cuidadores de síndrome demencial, Garrido e Menezes (2004) não encontraram associação entre a sobrecarga dos cuidadores e as variáveis sexo, idade, escolaridade e realização de atividades rotineiras de vida diária.

Dentre os cuidadores, a relação de parentesco agrupada em três grupos - cônjuge, filho e outros - não foi condição para resultados diferentes nos níveis de depressão no presente estudo. Achados similares foram apontados por Dura et al. (1991), que encontraram níveis de depressão e ansiedade semelhantes entre cuidadores cônjuges e filhos. Garrido e Menezes (2004) encontram que parentesco mais próximo ao doente, ou seja, ser cônjuge ou filho, associa-se a maiores níveis de impacto no cuidador, enquanto Conde-Sala et al. (no prelo) e Yeager et al. (2010) apontaram uma maior sobrecarga psicológica entre os filhos cuidadores. Considerando essas divergências, sugere-se a realização de estudos qualitativos e de caráter longitudinal que avaliem os diferentes impactos do cuidado para cônjuges, filhos e familiares com outros graus de parentesco com o doente.

No que se refere à presença de nível mais elevado de sintomas de depressão para cuidadores, os achados deste estudo concordam com outros que indicam ser a situação de cuidado de um familiar doente uma condição que pode contribuir para a ocorrência de depressão (Cuijpers, 2005; Pinquart \& Sörensen, 2003). A revisão sistemática de Schoenmakers, Buntinx, e De Lepeleire (no prelo) indica que a depressão ocorre em um de cada três cuidadores e é mais frequente naqueles que cuidam de pacientes com demência que em cuidadores de pacientes com outras doenças crônicas.

No presente estudo, comparando-se cuidadores e não 
cuidadores nos resultados das subescalas do BDI, destacouse a presença de mais sintomas de tipo cognitivo e afetivo da depressão para os cuidadores. Aspectos como tristeza, desânimo, desprazer, choro, irritação, diminuição de interesse pelas outras pessoas e dificuldade de tomar decisões mostraram-se mais elevados nessa população, o que sugere dificuldades para manter um senso pessoal de competência e autoconfiança quando as tarefas de cuidado são de difícil manejo. A depressão parece ser uma resposta psicológica à falta de controle sobre os sintomas do doente e, consequentemente, sobre as demandas do cuidado.

Por outro lado, observou-se resultados semelhantes na comparação entre cuidadores e não cuidadores do presente estudo em queixas relacionadas ao desempenho, a exemplo da capacidade de trabalhar, e somáticas, como diminuição de apetite e sono. Embora as diferenças entre cuidadores e não cuidadores se devam mais aos aspectos psicológicos como estresse, bemestar subjetivo, autoeficácia e depressão do que à saúde física, algumas pesquisas têm encontrado queixas somáticas e relatos de problemas de saúde entre cuidadores (Bodnar \& Kiecolt-Glaser, 1994; Goode et al., 1998; Ho et al., 2009; Pinquart \& Sörensen, 2003; Vitaliano et al., 2003).

Os resultados comparando cuidadores e não cuidadores nas subescalas de depressão sugerem que sintomas do tipo cognitivo e afetivo sejam mais preponderantes nessa população. A partir dos resultados encontrados de semelhança entre cuidadores e não cuidadores no fator Somático-Desempenho, é possível pensar que, em razão da necessidade de prontidão física diante das tarefas de cuidado, possa resultar mais difícil aos cuidadores qualificar os efeitos físicos gerados pela atividade de cuidado. Dessa forma, negligenciar eventuais condições precárias de sua saúde e considerar as condições de saúde do doente mais adversas que as próprias pode ser uma maneira de o cuidador lidar com a sobrecarga gerada em razão da necessidade de manutenção da intensa atividade de cuidado. Assim, recomenda-se que estudos futuros incluam a avaliação de estratégias de enfrentamento dos cuidadores e da presença de sintomas físicos diante da tarefa de cuidado.

$\mathrm{Na}$ amostra estudada, destacou-se o resultado de nível mais baixo de depressão para o grupo de idosos a partir de 65 anos. Sobre esse dado, é possível pensar na influência da participação em um grupo de apoio na redução de sintomas depressivos, considerando que a amostra a partir de 65 anos tanto de cuidadores quanto de não cuidadores foi recrutada, em especial, a partir de grupos de apoio e de convivência, respectivamente. Dessa forma, a influência dessa variável deve ser mais bem detalhada em estudos futuros.

Também com relação a esse resultado, é possível que a escala utilizada não esteja sendo sensível para avaliar a depressão na população idosa, tal como Mann (2001) sugeriu a respeito da não adequação de alguns instrumentos para medir depressão em idosos por não contemplarem manifestações clínicas mais típicas dessa faixa etária. No que se refere ao instrumento de medida utilizado neste estudo, o manual brasileiro do Inventário Beck de Depressão (Cunha, 2001), indica que estudos revisados envolvendo o inventário não encontraram relação significativa entre os escores de depressão e a idade. Entretanto, recomendase que sejam controladas variáveis psicossociais cuja correlação com os escores do BDI superem 0,30. Portanto, considerando-se o resultado encontrado no presente estudo de correlação negativa significativa entre escores de depressão e idade, sugere-se a realização de pesquisas com outros instrumentos específicos para avaliação de idosos, por exemplo, a Center Epidemiologic Survey - Depression (CES-D) de Radloff (1977), validada no Brasil para a população idosa por Tavares (2004). Também poderiam ser aplicadas a Escala Geriátrica de Depressão (Geriatric Depression Scale - GDS), de Yesavage et al. (1983), cuja versão de 15 itens foi validada no Brasil por Almeida e Almeida (1999) e, segundo Tumas, Rodrigues, Farias, e Crippa (2008), mostrou-se melhor que o BDI para avaliar a precisão do diagnóstico de depressão em pacientes com doença de Parkinson. Além disso, poderiam ser realizados estudos com a segunda versão do Inventário Beck de Depressão (Beck, Steer, \& Brown, 1996), em fase de adaptação no Brasil (Wendt, Rusch, Mallet, \& Argimon, 2008), para averiguar a hipótese de baixa sensibilidade do BDI para avaliação de depressão em idosos.

Também relevantes neste estudo foram os resultados mais elevados de depressão para cuidadores, em especial da faixa etária de 56 a 64 anos, em que aparece uma diferença importante quando comparada a não cuidadores nas características do Fator Cognitivo-Afetivo. Esses achados contrariam os dados encontrados por Sanders e Adams (2005) que verificaram maiores níveis de depressão entre os cuidadores mais jovens, filhos dos doentes, sendo que, no referido estudo, a média de idade foi de 59 anos com variação ente 28 e 89 anos. Contudo, a meta-análise com 84 estudos realizada por Pinquart e Sörensen (2003) identificou que amostras compostas por cuidadores mais velhos apresentavam significativamente maiores níveis de depressão quando comparados aos mais jovens. Especificamente quanto à avaliação de depressão, em um estudo brasileiro com pessoas idosas, Fortes-Burgos, Neri, e Cupertino (2008) encontraram uma associação de maior risco de ocorrência de depressão na faixa etária de 60 a 69 anos em uma amostra dividida por intervalos de idade que variaram de 60 a 69 anos, de 70 a 79 anos, e de 80 a 99 anos de idade. O estudo indicou menores níveis de depressão para pessoas com mais idade nessa amostra, corroborando os dados relacionados à idade do presente estudo.

Considerando estes aspectos, é possível pensar que, dentre os cuidadores do presente estudo, aqueles com idades entre 56 e 64 anos encontravam-se lidando com demandas provenientes de outros papéis sociais, por exemplo, família e trabalho, que se somavam às responsabilidades com o familiar doente, causando-lhes maior sobrecarga emocional, em especial em termos cognitivos e afetivos. Concernente a isso, o estudo de Conde-Sala et al. (no prelo) indicou que a sobrecarga tende a ser maior para cuidadores que são filhos do doente, que vivem com o paciente e que possuem outras obrigações familiares. No que se refere ao presente estudo, também é possível pensar que os cuidadores mais jovens e os mais velhos recebessem o apoio de outros familiares na tarefa de cuidado, possivelmente teriam mais recursos psicológicos e em sua rede social para enfrentar a doença do seu familiar.

A partir desta pesquisa foi possível verificar que cuidar de um familiar portador de síndrome demencial pode mobilizar 
intensamente os recursos emocionais do cuidador frente às demandas que a sintomatologia e o curso da doença impõem. Quando estas demandas excedem os recursos psicológicos individuais, os cuidadores tendem a apresentar um grande desgaste emocional, manifestando, como exemplo da amostra estudada, um nível mais elevado de sintomas afetivos e cognitivos relacionados à depressão. Assim, considera-se fundamental a atenção à saúde psicológica de cuidadores, bem como o fortalecimento da rede de apoio para o auxílio na atividade de cuidado. Sugerem-se estudos que avaliem a presença de depressão por meio de outros instrumentos e a influência da idade com o controle de outras variáveis, por exemplo, demais demandas diárias. Também é indicada a avaliação do apoio social recebido pelo cuidador, aspecto que, dependendo da ausência ou presença e do tipo de apoio recebido (Gonçalves, Pawlowski, Bandeira, \& Piccinini, no prelo), pode contribuir para o agravamento ou a diminuição do impacto gerado pela tarefa de cuidado.

\section{Referências}

Almeida, O. P., \& Almeida, S. A. (1999). Short versions of the Geriatric Depression Scale: a study of their validity for the diagnosis of a major depressive episode according to ICD-10 and DSM-IV. International Journal of Geriatric Psychiatry, 14, 858-865.

Anthony-Bergstone, C. R., Zarit, S. H., \& Gatz, M. (1988). Symptoms of psychological distress among caregivers of dementia patients. Psychology and Aging, 3(3), 245-248.

Bandeira, D. R., Pawlowski, J., Gonçalves, T. R., Hilgert, J. B., Bozzetti, M. C., \& Hugo, F. N. (2007). Psychological distress in Brazilian caregivers of relatives with dementia. Aging \& Mental Health, 11(1), 14-19.

Bauer, M. E., Vedhara, K., Perks, P., Wilcok, G. K., Lightman, S. L., \& Shanks, N. (2000). Chronic stress in caregivers of dementia patients is associated whit reduced lymphocyte sensitivity to glucocorticoids. Journal Neuroimmunology, 103, 84-92.

Beck, A. T., \& Steer, R. A. (1993). Manual for the Beck Depression Inventory. San Antonio: The Psychological Corporation.

Beck, A. T., Steer, R. A., \& Brown, G. K. (1996). BDI-II. Beck Depression Inventory-second edition. Manual. San Antonio: The Psychological Corporation.

Bodnar, J. C., \& Kiecolt-Glaser, J. K. (1994). Caregiver depression after bereavement: chronic stress isn't over when it's over. Psychology and Aging, 9(3), 372-380.

Cacioppo, J. T., Poehlmann, K. M., Kiecolt-Glaser, J. K., Malarkey, W. B., Burleson, M. H., ... \& Glaser, R. (1998). Cellular immune responses to acute stress in female caregivers of dementia patients and matched controls. Health Psychology, 17(2), 182-189.

Christensen, H., Jorm, A. F., Mackinnon, A. J., Korten, A. E., Jacomb, P. A., ... \& Rodgers, B. (1999). Age differences in depression and anxiety symptoms: a structural equation modeling analysis of data from a general population sample. Psychological Medicine, 29(2), 325-339.

Conde-Sala, J. L., Garre-Olmo, J., Turró-Garriga, O., Vilalta-Franch, J., \& LópezPousa, S. (no prelo). Differential features of burden between spouse and adult-child caregivers of patients with Alzheimer's disease: an exploratory comparative design. International Journal of Nursing Studies.

Cuijpers, P. (2005). Depressive disorders in caregivers of dementia patients: a systematic Review. Aging \& Mental Health, 9(4), 325-330.

Cunha, J. A. (2001). Escalas Beck. São Paulo: Casa do Psicólogo.

Dura, J. R., Stukenberg, K. W., \& Kiecolt-Glaser, J. K. (1990). Chronic stress and depressive disorders in older adults. Journal of Abnormal Psychology,
99(3), 284-290.

Dura, J. R., Stukenberg, K. W., \& Kiecolt-Glaser, J. K. (1991). Anxiety and depressive disorders in adult children caring for demented parents. Psychology and Aging, 6(3), 467-473.

Ferrara, M., Langiano, E., Brango, T., Vito, E., Cioccio, L., \& Bauco, C. (2008) Prevalence of stress, anxiety and depression in with Alzheimer caregivers. Health and Quality of Life Outcomes, 6(93), Recuperado de http://www. ncbi.nlm.nih.gov/pmc/issues/161304/

Fortes-Burgos, A. C. G., Neri, A. L., \& Cupertino, A. P. F. B. (2008). Eventos estressantes, estratégias de enfrentamento, auto-eficácia e sintomas depressivos entre idosos residentes na comunidade. Psicologia: Reflexão e Critica, 21(1), 74-82.

Garrido, R., \& Menezes, P. R. (2004). Impacto em cuidadores de idosos com demência atendidos em um serviço psicogeriátrico. Revista de Saúde Pública, 38(6), 835-841.

Gaugler, J. E., Davey, A., Pearlin, L. I., \& Zarit, S. H. (2000). Modeling caregiver adaptation over time: the longitudinal impact of behavior problems. Psychology \& Aging, 15(3), 437-450.

Gazalle, F. K., Lima, M. S., Tavares, B. K., \& Hallal, P. C. (2004). Sintomas depressivos e fatores associados em população idosa no Sul do Brasil. Revista de Saúde Pública, 38(3), 365-371.

Gonçalves, T. R., Pawlowski, J., Bandeira, D. R., \& Piccinini, C. A. (no prelo). Avaliação de apoio social em estudos brasileiros: aspectos conceituais e instrumentos. Ciência \& Saúde Coletiva. Recuperado de http://www. cienciaesaudecoletiva.com.br

Goode, K. T., Haley, W. E., Roth, D. L., \& Ford, G. R. (1998). Predicting longitudinal changes in caregiver physical and mental health: a stress process model. Health Psychology, 17(2), 190-198.

Ho, S. A., Chan, A., Woo, J., Chong, P., \& Sham, A. (2009). Impact of caregiving on health and quality of life: a comparative population-based study of caregivers for elderly persons and noncaregivers. The Journals of Gerontology Series A: Biological Sciences and Medical Sciences, 64A(8), 873-879.

Jorm, A. F. (2000). Does old age reduce the risk of anxiety and depression? A review of epidemiological studies across the adult life span. Psychological Medicine, 30(1), 1-22.

Leite, V. M. M., Carvalho, E. M. F., Barreto, K. M. L, \& Falcão, I. V. (2006) Depressão e envelhecimento: estudo nos participantes do Programa Universidade Aberta à Terceira Idade. Revista Brasileira de Saúde MaternoInfantil, 6(1), 31-28.

Mann, A. (2001). Depression in the elderly: findings from a community survey. Maturitas, 38(1), 53-59.

Penninx, B. W., Deeg, D. J., van Eijk, J. T., Beekman, A. T., \& Guralnik, J. M. (2000). Changes in depression and physical decline in older adults: a longitudinal perspective. Journal of Affective Disorders, 61(1), 1-12.

Pinquart, M., \& Sörensen, S. (2003). Differences between caregivers and noncaregivers in psychological health and physical health: a meta analysis. Psychology and Aging, 18(2), 250-267.

Plati, M. C. F., Covre, P., Lukasova, K., \& Macedo, E. C. (2006). Depressive symptoms and cognitive performance of the elderly: relationship between institutionalization and activity programs. Revista Brasileira de Psiquiatria, 28(2), 118-121.

Radloff, L. S. (1977). The CES-D scale: a self-report depression scale for research in the general population. Applied Psychological Measurement, 1, 385-401.

Russo, J., Vitaliano, P. P., Brewer, D. D., Katon, W., \& Becker, J. (1995). Psychiatric disorders in spouse caregivers of care recipients with Alzheimer's disease and matched controls: a diathesis-stress model of psychopathology. Journal of Abnormal Psychology, 104(1), 197-204.

Sanders, S., \& Adams, K. B. (2005). Grief reactions and depression in caregivers of individuals with Alzheimer's disease:results from a pilot study in an urban setting. Health \& Social Work, 30(4), 287-295.

Schoenmakers, B., Buntinx, F., \& De Lepeleire, J. (2009). The relation between care giving and the mental health of caregivers of demented relatives: a cross- 
sectional study. The European Journal of General Practice, 15(2), 99-106.

Schoenmakers, B., Buntinx, F., \& De Lepeleire, J. (no prelo). Factors determining the impact of care-giving on caregivers of elderly patients with dementia: a systematic literature review. Maturitas.

Schoevers, R. A., Beeckman, A. T. F., Deeg, D. J. H., Geerlings, M. I., Jonker, C., \& van Tilburg, W. (2000). Risk factors for depression in later life: results of a prospective community based study (AMSTEL). Journal of Affective Disorders, 59(1), 127-137.

Snowdon, J. (2002). How high is the prevalence of depression in old age? [Suplemento 1]. Revista Brasileira de Psiquiatria, 24, 42-47.

Stordal, E., Mykletun, A., \& Dahl, A. A. (2003). The association between age and depression in the general population: a multivariate examination. Acta Psychiatrica Scandinavica, 107, 132-141.

Tavares, S. S. (2004). Sintomas depressivos entre idosos: relações com classe, mobilidade e suporte social percebidos e experiência de eventos estressantes. (Dissertação de Mestrado, Universidade Estadual de Campinas, Campinas).

Trentini, C. M., Xavier, F. M. F., Chachamovich, E., Rocha, N. S., Hirakata, V. N., \& Fleck, M. P. A. (2005). The influence of somatic symptoms on the performance of elders in the Beck Depression Inventory (BDI). Revista Brasileira de Psiquiatria, 27(2), 119-123.
Tumas, V., Rodrigues, G. G. R., Farias, T. L. A., \& Crippa, J. A. S. (2008). The accuracy of diagnosis of major depression in patients with parkinson's disease: a comparative study among the UPDRS, the Geriatric Depression Scale and the Beck Depression Inventory. Arquivos de Neuropsiquiatria, 66(2-A), 152-156.

Vitaliano, P. P., Russo, J. Young, H. M., Teri, L., \& Maiuro, R. D. (1991). Predictors of burden in spouse caregivers of individuals with Alzheimer's disease. Psychology and Aging, 6(3), 392-402.

Vitaliano, P. P., Zhang, J., \& Scanlan, J. M. (2003). Is caregiving hazardous to one's physical health? A meta-analysis. Psychological Bulletin, 129, 946-972.

Wendt, G. W., Rusch, S. G. S., Mallet, L. G., \& Argimon, I. I. L. (2008). Adaptação do inventário de depressão de Beck-II (BDI-II) para a população brasileira. In IX Salão de Iniciação Científica da PUCRS. Anais (pp. 1-3). Porto Alegre: EDIPUCRS

Yeager, C. A., Hyer, L. A., Hobbs, B., \& Coyne, A. C. (2010). Alzheimer's disease and vascular dementia: the complex relationship between diagnosis and caregiver burden. Issues in Mental Health Nursing, 31(6), 376-384.

Yesavage, J. A., Brink, T. L., Rose, T. L., Lum, O., Huang, V., ... \& Leirer, V. O. (1983). Development and validation of a geriatric depression screening scale: a preliminary report. Journal of Psychiatric Research, 17(1), 37-49.

Josiane Pawlowski, mestre em Psicologia pela Universidade Federal do Rio Grande do Sul (UFRGS), é doutoranda em Psicologia na Pós-Graduação em Psicologia da mesma instituição. Endereço para correspondência: Universidade Federal do Rio Grande do Sul, Instituto de Psicologia. Rua Ramiro Barcelos, 2600, sala 120, Bairro Santa Cecília, CEP 90035-003, Porto Alegre, RS, Brasil. Telefone: (51) 3308-5352. E-mail: josipski@yahoo.com.br

Tonantzin Ribeiro Gonçalves, mestre em Psicologia pela Universidade Federal do Rio Grande do Sul (UFRGS), é doutoranda em Psicologia da mesma instituição. E-mail: tonanrib@yahoo.com.br

Juliana Balbinot Hilgert, doutora em Epidemiologia pela Universidade Federal do Rio Grande do Sul (UFRGS), é professora adjunta do Departamento de Odontologia Preventiva e Social da Faculdade de Odontologia da mesma instituição. E-mail: jhilgert@gmail.com

Fernando Neves Hugo, doutor em Odontologia: Saúde Coletiva pela Universidade Estadual de Campinas (UNICAMP), é professor adjunto do Departamento de Odontologia Preventiva e Social da Faculdade de Odontologia da Universidade Federal do Rio Grande do Sul (FO-UFRGS). E-mail: fernandoneveshugo@ gmail.com

Mary Clarisse Bozzetti, doutora em Medicina: Ciências Médicas pela Universidade Federal do Rio Grande do Sul (UFRGS), é professora associada II do Departamento de Medicina Social da Faculdade de Medicina da mesma instituição. E-mail: mcbozzetti@yahoo.com.br

Denise Ruschel Bandeira, doutora em Psicologia pela Universidade Federal do Rio Grande do Sul (UFRGS), é professora adjunta do Departamento de Psicologia do Desenvolvimento e da Personalidade do Instituto de Psicologia da mesma instituição. E-mail: drbandei@terra.com.br 